\title{
Comparative analysis of the ability of Clostridium clariflavum strains and Clostridium thermocellum to utilize hemicellulose and unpretreated plant material
}

\author{
Javier A Izquierdo ${ }^{1,2,3}$, Sivakumar Pattathil ${ }^{2,4}$, Anna Guseva ${ }^{1,2}$, Michael G Hahn $n^{2,4}$ and Lee R Lynd ${ }^{1,2^{*}}$
}

\begin{abstract}
Background: Among themophilic consolidated bioprocessing (CBP) candidate organisms, environmental isolates of Clostridium clariflavum have demonstrated the ability to grow on xylan, and the genome of C. clariflavum DSM 19732 has revealed a number of mechanisms that foster solubilization of hemicellulose that are distinctive relative to the model cellulolytic thermophile Clostridium thermocellum.

Results: Growth experiments on xylan, xylooligosaccharides, and xylose reveal that $C$. clariflavum strains are able to completely break down xylan to xylose and that the environmental strain C. clariflavum sp. 4-2a is able to grow on monomeric xylose. C. clariflavum strains were able to utilize a larger proportion of unpretreated switchgrass, and solubilize a higher proportion of glucan, xylan, and arabinan, with strain 4-2a reaching the highest extent of solubilization of these components (64.7 to $69.4 \%$ ) compared to C. thermocellum (29.5 to $42.5 \%$ ). In addition, glycome immunoanalyses of residual plant biomass reveal differences in the extent of degradation of easily accessible xylans, with C. clariflavum strains having increased solubilization of this fraction of xylans relative to C. thermocellum.
\end{abstract}

Conclusions: C. clariflavum strains exhibit higher activity than C. thermocellum in the breakdown of hemicellulose and are capable of degrading xylan to xylooligomers and xylose. This capability seems to also play a role in the higher levels of utilization of unpretreated plant material.

Keywords: CBP, Clostridium thermocellum, Clostridium clariflavum, Hemicellulose, Switchgrass

\section{Background}

Consolidated bioprocessing, or CBP, is one of the most promising and widely recognized strategies for the microbial conversion of lignocellulosic biomass into products of interest [1], with thermophilic, cellulolytic bacteria often considered in this context. It is curious to note, however, that the model thermophile Clostridium thermocellum and several of its close relatives do not grow on hemicellulose or its component pentose sugars. Indeed, the only moderately thermophilic (optimal growth temperature about $60^{\circ} \mathrm{C}$ ) cellulolytic anaerobes we know of that grow on xylose, xylooligomers, or other hemicellulose components are an

\footnotetext{
* Correspondence: lee.lynd@dartmouth.edu

'Thayer School of Engineering, Dartmouth College, Hanover, NH, USA ${ }^{2}$ BioEnergy Science Center Oak Ridge National Laboratory Oak Ridge, Oak Ridge, TN, USA

Full list of author information is available at the end of the article
}

environmental isolate of C. clariflavum previously reported by our group [2] and a distant relative of these clostridia, C. stercorarium [3]. Other clostridia from the same phylogenetic cluster (Cluster III), such as C. cellulolyticum, can utilize both cellulose and hemicellulose, but are able to do so at mesophilic temperatures, which results in slower fermentation rates [4].

The type strain of Clostridum clariflavum, DSM 19732, was originally isolated from anaerobic sludge and is a very close relative of fellow cellulolytic thermophiles within Clostridia Cluster III, C. thermocellum and C. straminisolvens [5,6]. Much like C. thermocellum, this organism employs a cellulosome to break down plant biomass and can grow very effectively on cellulose but not on hemicellulose. In an effort to expand the known metabolic diversity of this species, we have previously described the 
enrichment and isolation of novel C. clariflavum strains from thermophilic compost, and reported on their ability to grow on xylan, which is a key differentiating capability compared to DSM 19732 [2,7]. In addition, the genome of C. clariflavum DSM 19732 reveals a very distinct mechanism for the breakdown of hemicellulose, as compared to its close relative C. thermocellum [8].

In the work reported here, we explore and characterize the ability of two representative C. clariflavum strains, the type strain DSM 19732 and environmental isolate strain 42a, to utilize hemicellulose, both in the form of C5 monomers and polymers (xylose, xylooligosaccharides, xylan) and in unpretreated plant material, and compare these characteristics to those of $C$. thermocellum.

\section{Results}

\section{Dynamics of xylan utilization}

The dynamics of xylan hydrolysis were examined for $C$. clariflavium strains DSM 19732 and 4-2a as well as C. thermocellum. All three strains exhibited different xylan hydrolysis and utilization behaviors (Figure 1). C. thermocellum inocula were able to hydrolyze xylan to xylooligomers (such as the xylotriose detected in this experiment), but were not able to grow on xylan or produce any detectable xylose (Figure 1A). C. clariflavum DSM 19732 was able to release xylooligosaccharides from the xylan substrate that were quickly broken down to xylose, but was also not able to grow on these substrates (Figure 1B). Hydrolysis of xylan and xylooligosaccharides to xylose slowly continued beyond 144 hours, resulting in a final measurement of $0.3 \mathrm{~g}$ xylose/L. Both C. thermocellum and C. clariflavum DSM 19732 experiments provide evidence of enzymatic breakdown of xylan by the inocula utilized, but no growth. C. clariflavum str. 4-2a was able to grow on $3 \mathrm{~g} / \mathrm{L}$ xylan (Figure $1 \mathrm{C}$ ). Xylooligosaccharides (in the form of xylotriose) were observed early in the fermentation process, but appear to be quickly hydrolyzed all the way to xylose. Some residual xylose was not utilized by C. clariflavum str. 4-2A and accumulates to a final concentration of $0.12 \mathrm{~g} / \mathrm{L}$. In addition to turbidity and microscopic examination indicative of cell growth, formation of acetic acid and formic acid by str. 4-2A, but not other strains, provided evidence of fermentation.

\section{Dynamics of xylooligosaccharide utilization}

C. clariflavum strain 4-2a was able to grow on all three xylooligosaccharides (xylobiose, xylotriose, and xylotetraose) we tested (Figure 2), with similar behavior as observed for xylan. For all three xylooligosaccharides, larger molecules were completely broken down into smaller components within the first 24 hours of growth. Xylose that resulted from xylooligosaccharide hydrolysis was slowly fermented over 100 to 120 hours, but eventually was completely utilized (Figure 2A, B, C). Acetic acid production was detected at the same time as xylose utilization was observed. In comparison, C. clariflavum strain DSM 19732 was able to break down all xylooligosaccharides to xylooligosaccharides of smaller size and eventually all the way to xylose, but it was not able to utilize the resulting xylose, which resulted in accumulation of xylose in growth experiments using all three xylooligosaccharides (Figure 2D, E, F). The lack of fermentation products (acetate) further confirms the inability of this organism to utilize xylose resulting from xylooligosaccharide hydrolysis. No growth was observed in C. thermocellum ATCC 27405 cultures growing on the three xylooligosaccharides tested.

\section{Dynamics of xylose utilization}

C. clariflavum strain 4-2a was able to grow on xylose as the sole carbon source (Figure 3). Evidence of fermentation was provided by the detection of acetate and formate between 48 and 72 hours after inoculation. Likewise, evidence of cell growth was provided by the observed
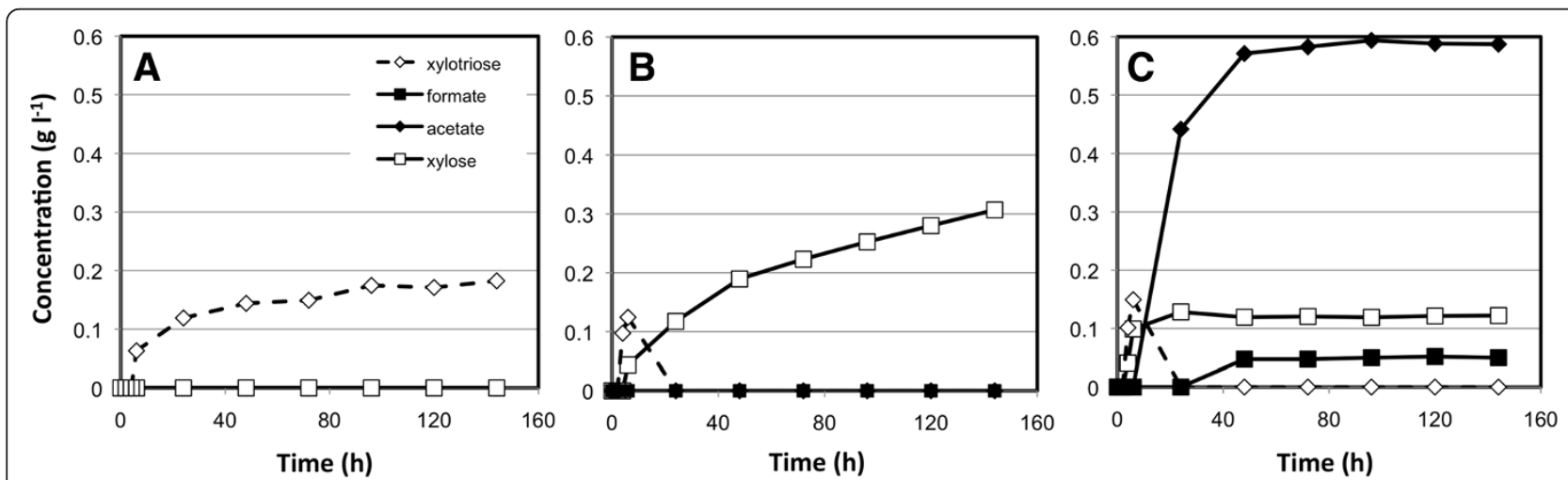

Figure 1 Dynamics of soluble product formation from xylan growth experiments. The dynamics of products of xylan hydrolysis (xylotriose and xylose) and fermentation products (acetate, formate) are shown for growth experiments for C. thermocellum ATCC 27405 (A), C. clariflavum DSM 19732 (B), and C. clariflavum str. 4-2a (C), using xylan as the only carbon source. 

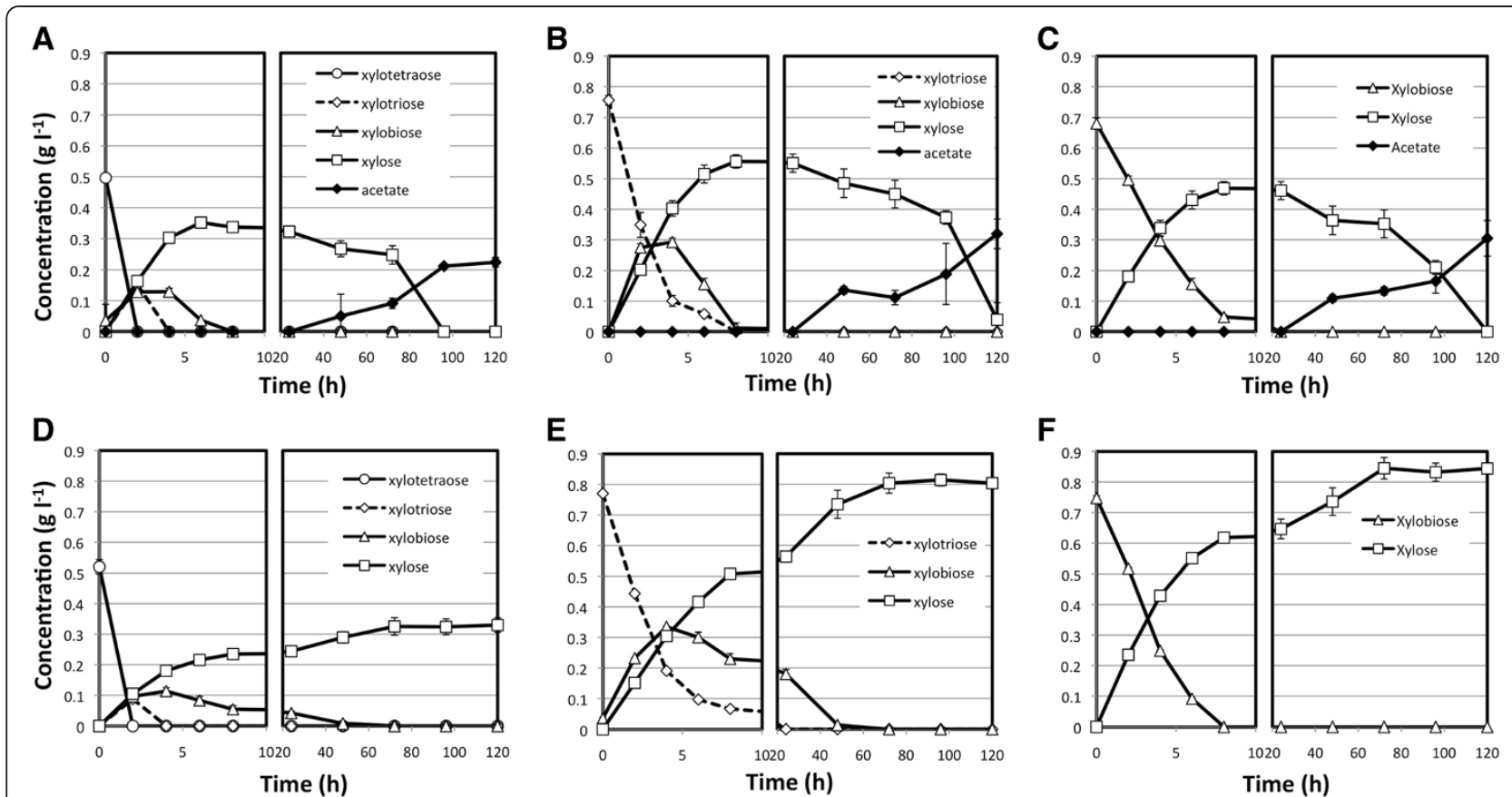

E

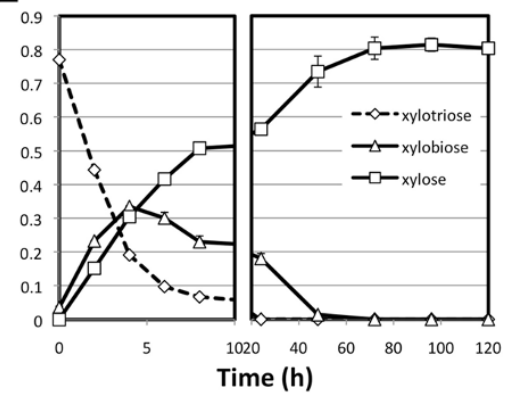

$\mathbf{F}$



Figure 2 Dynamics of xylooligosaccharide utilization for $C$. clariflavum strains. The breakdown dynamics and fermentation product formation are shown for triplicate experiments using xylotetraose (A, D), xylotriose (B, E), and xylobiose (C, F), for growth experiments inoculated with C. clariflavum str. 4-2a (A, B, C) and C. clariflavum DSM 19732 (D, E, F).

increased cell mass and total pellet nitrogen content over the first 96 hours of culture, which was followed by a decline thereafter. Complete utilization of $2.5 \mathrm{~g} / \mathrm{L}$ of xylose was slow, taking approximately 120 hours. However, and by comparison, $C$. thermocellum ATCC 27405 and

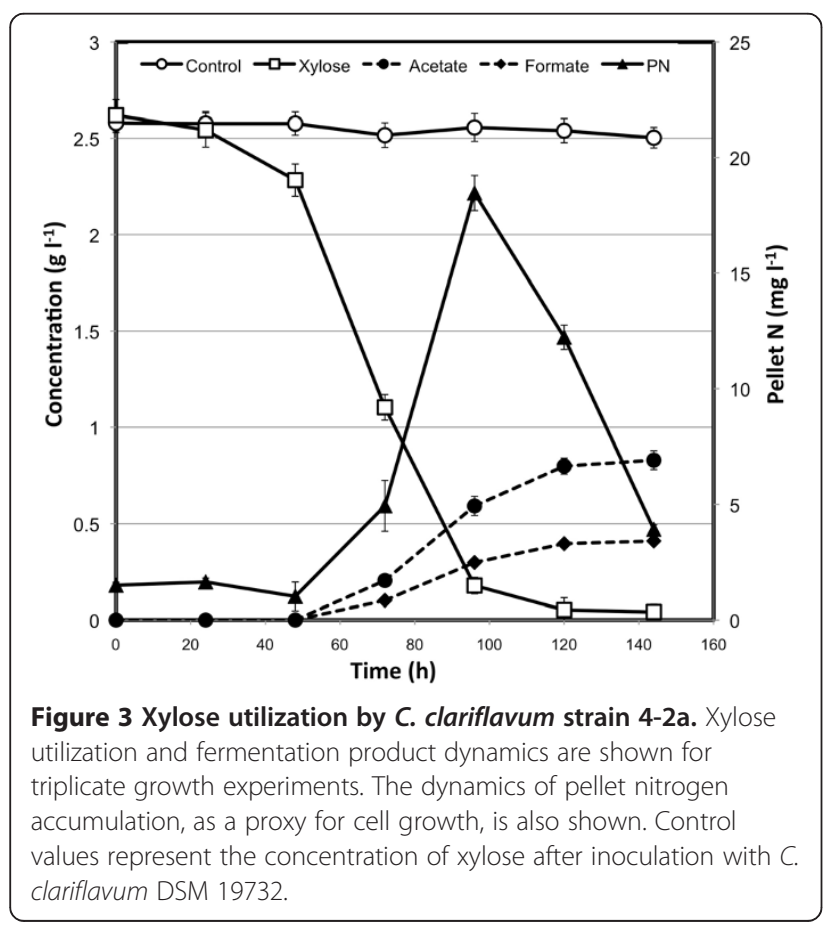

C. clariflavum DSM 19732 were not able to grow on $x y-$ lose, as demonstrated by the lack of xylose utilization in the control experiment with C. clariflavum DSM 19732. Similar behavior was observed in bottle experiments with C. thermocellum ATCC 27405 (data not shown).

\section{Conversion of unpretreated switchgrass}

After 5 days of incubation on switchgrass without pretreatment beyond autoclaving, batch fermentations with C. clariflavum str. 4-2a contained residual plant material averaging $41.2 \pm 1.4 \%$ relative to the weight of the original plant material (58.8\% solubilization). C. clariflavum DSM 19732 and C. thermocellum ATCC 27405 fermentations contained $57.6 \pm 4.1 \%$ (42.4\% conversion) and $66.5 \pm 1.5 \%$ (32.5\% conversion), respectively (Figure $4 \mathrm{~A})$. A set of uninoculated controls displayed little weight loss after incubation at $55^{\circ} \mathrm{C}$, with $97.9 \pm 0.8 \%$ of the original plant material being accounted for. Similarly, residual glucan, xylan, and arabinan contents measured by quantitative saccharification in all of these experiments were higher after inoculation with $C$. thermocellum than with C. clariflavum strains (Figure 4B). Quantitative saccharification to determine the sugar composition of uninoculated switchgrass controls resulted in a baseline composition of $35.5 \pm$ $0.7 \%$ glucan, $24.5 \pm 0.6 \%$ xylan, and $3.5 \pm 0.2 \%$ arabinan. Conversion percentages were calculated using these values and the residual glucan, xylan, and arabinan measured in the inoculated experiments. As seen in Figure 4B, 
A

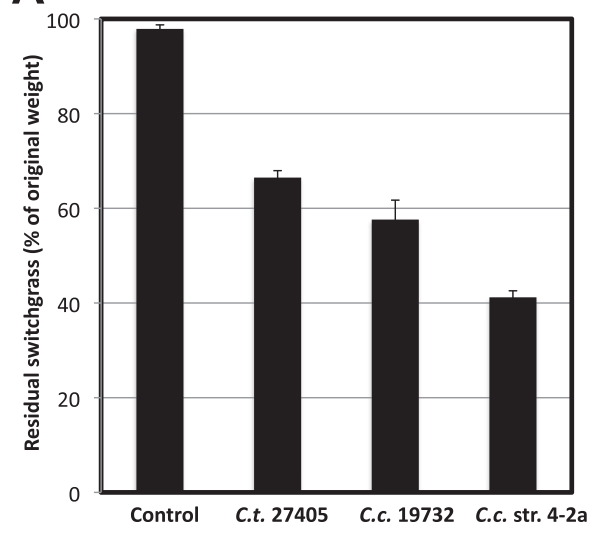

B

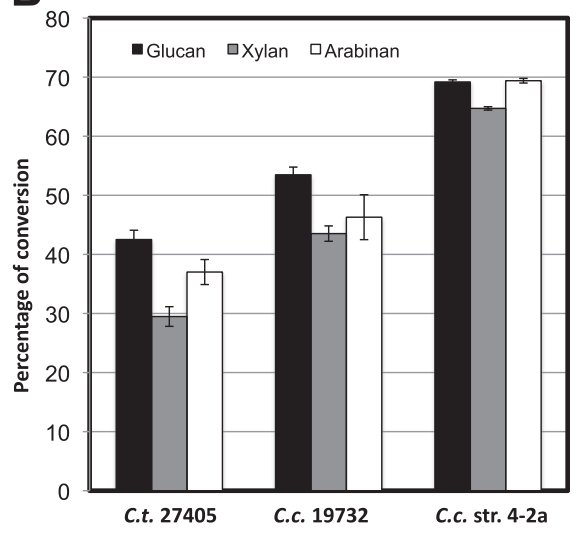

Figure 4 Unpretreated switchgrass utilization by $C$. thermocellum and C. clariflavum strains. Percentage of residual switchgrass relative to original weight (A) is shown for triplicate experiments with C. thermocellum ATCC 27405, C. clariflavum DSM 19732, C. clariflavum str. 4-2a, and uninoculated controls after 5 days of incubation. Percentage of glucan, xylan, and arabinan utilization (B) is also shown for all three organisms.

C. clariflavum str. 4-2a was the most efficient of the three strains in terms of conversion of switchgrass components, reaching between $64.7 \%$ (xylan) and $69.4 \%$ (arabinan) conversion. Also, C. clariflavum DSM 19732 is more efficient than C. thermocellum ATCC 27405, breaking down all three components with conversion percentages in the 43.5 to $53.5 \%$ range compared to 29.5 to $42.5 \%$ in C. thermocellum (Figure 4B).

\section{Switchgrass glycomics}

To obtain further insights into cell wall glycan utilization during microbial growth on switchgrass biomass, glycome profiling analyses were conducted on untreated switchgrass (original plant material), uninoculated switchgrass controls (original plant material incubated in media without bacteria), and switchgrass incubated with microbial strains, C. thermocellum ATCC 27405, C. clariflavum DSM 19732, and C. clariflavum str. 4-2a (Figure 5). First, two notable differences were observed in the glycome profiles of uninoculated controls compared to those of untreated switchgrass. The most notable such difference was the significantly higher abundance of xylan epitopes recognized by the xylan- 4 through -7 groups of monoclonal antibodies (mAbs) in the oxalate and carbonate extracts obtained from uninoculated controls compared to those of untreated residues. In addition, there was an increased abundance of pectic-arabinogalactan epitopes in nearly all extracts isolated from uninoculated controls in comparison to untreated switchgrass. Interestingly, the glycome profiling analyses revealed distinct changes in overall cell wall glycan composition and extractability in switchgrass residues that had been incubated with any of the microbial strains in comparison to those of uninoculated controls. All such differences are indicated with green and blue squares in Figure 5. In general, an overall reduction in the abundance of xylan epitopes (as indicated by the reduced binding intensities of xylan-4, xylan-5, xylan-6, and xylan7 groups of mAbs) was apparent in the oxalate and carbonate extracts isolated from switchgrass residues that had been incubated with any of the three microbial strains in comparison to those of uninoculated controls. Indeed, almost all of the carbonate extractable xylan that was immunologically detectable was absent in the microbially digested wall residues. However, in the oxalate extracts, both $C$. clariflavum strains exhibited increased depletion of xylan epitopes (as indicated by the reduced intensities of mAb binding) compared to that of the C. thermocellum strain. In general, the glycome profiles of microbeincubated biomass did not exhibit any significant variations in the case of the other alkaline and chlorite extracts (harsh extracts). Some subtle differences were observed among extracts isolated from microbe-incubated biomass, such as a depletion of $1 \mathrm{M} \mathrm{KOH}$-released xyloglucan epitopes and a marginal reduction in some $4 \mathrm{M} \mathrm{KOH}-$ released xyloglucan epitopes, and a small increase in the abundance of $\beta$-glucan epitopes in $1 \mathrm{M} \mathrm{KOH}$ and $4 \mathrm{M}$ $\mathrm{KOH} \mathrm{PC} \mathrm{extracts.} \mathrm{Overall,} \mathrm{the} \mathrm{glycome} \mathrm{profiling} \mathrm{studies}$ reveal a preferential utilization of loosely associated hemicellulosic xylans in switchgrass biomass by all three microbial strains studied, with C. clariflavum strains being more efficient in terms of xylan solubilization.

\section{Discussion}

In this work we have tested and demonstrated the ability of C. clariflavum strains to utilize hemicellulose as it relates to the model organism and close relative, $C$. thermocellum. At various levels of complexity (xylose, xylooligosaccharides, xylan, and unpretreated plant material), hemicellulose breakdown was markedly different between $C$. thermocellum and C. clariflavum strains. Compared to $C$. thermocellum, our results indicate that C. clariflavum strains have a distinctive ability to break 


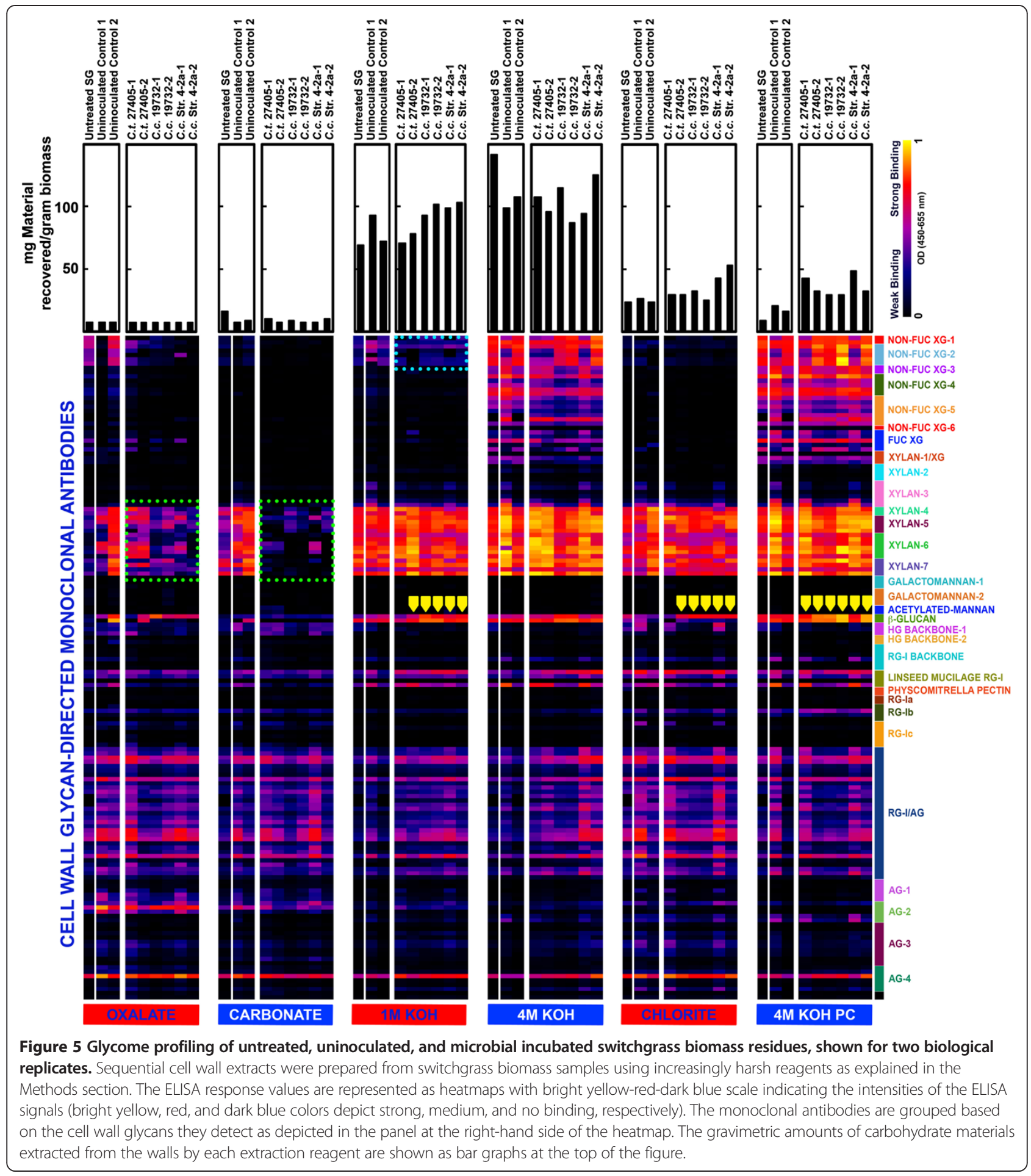

down xylan into smaller xylooligosaccharides all the way to xylose within a very short amount of time. Our results also make it evident that this is an inherent enzymatic capability of C. clariflavum strains, regardless of whether they are able to grow on xylose or not.

A key differentiating factor for C. clariflavum strain 4-2a relative to the two other strains examined is its ability to utilize xylose for growth. In all experiments using xylan, xylooligosaccharides, and xylose as sole carbon sources, it was possible to confirm the partial to full utilization of the 5-carbon monomer, although at a much slower rate than the rate of oligomer solubilization. Also, comparatively, $C$. clariflavum strains DSM 19732 and 4-2a grow much faster on cellobiose than the growth displayed by strain $4-2 \mathrm{a}$ on 
xylose. Xylose isomerase and xylulose kinase are both essential genes for the utilization of xylose in Clostridia [9]. Neither gene is found in C. thermocellum, and only xylulose kinase is present in C. clariflavum DSM 19732 [8]. The genome of strain 4-2a has recently been completed, and we have been able to find a gene for xylose isomerase to be present in this strain, along with the xylulose kinase gene already detected in DSM 19732. This largely explains our observations of xylose utilization by strain 4-2a, as compared to DSM 19732 and C. thermocellum. There are also key differences in the $\beta$-xylosidases found in C. thermocellum versus those found in C. clariflavum. For example, the GH39 B-xylosidase present in C. clariflavum is closely related to similar enzymes in xylanolytic Clostridia that include C. stercorarium BxlB, an enzyme demonstrated to break down xylooligomers to xylose [10], and a xylosidase found in Clostridium sp. DL-VIII [11], whereas the one found in C. thermocellum has sequence homology to xylosidases found in more distantly related actinomycetes. Similarly, most of the GH43 $\beta$-xylosidases found in the $C$. clariflavum genome have no homologs among the fewer representatives found in the $C$. thermocellum genome.

An increasing number of organisms have been successfully discovered and developed to utilize both cellulose and hemicellulose polymers and sugars [12-14]. However, one limitation identified in many of these cases has been the need to use yeast extract as part of the growth medium for these organisms to be able to grow on these complex substrates $[15,16]$. We have observed, in previous work [2] and in the work reported in this paper, that one feature of C. clariflavum species is their demonstrated capability to grow on cellulose and hemicellulose components of plant biomass in defined media without the addition of yeast extract. This should represent a beneficial feature of this organism if it is to be developed for larger scale processes.

C. clariflavum strains demonstrated a higher extent of conversion of unpretreated switchgrass than did C. thermocellum, both in terms of total conversion (net residual weight) and the ability to break down polymers containing glucan, xylan, and arabinan, with strain 4-2a exhibiting the highest conversion of the three strains examined. In terms of net residual weight, all three clostridial species seem to utilize unpretreated switchgrass extensively (33 to $59 \%$ solubilization). Similarly, extreme thermophiles that are also able to utilize both cellulose and hemicellulose, such as Caldicellulosiruptor bescii, have been reported to achieve 20 to $25 \%$ conversion of unpretreated switchgrass $[14,16]$. C. bescii has been shown to further utilize material after successive reinoculations and fermentations, which was not part of our study with the three Clostridia discussed in this paper.

Glycome profiling of the utilized switchgrass also revealed higher efficiency in breaking down various xylan fractions by $C$. clariflavum strains as compared to $C$. thermocellum. C. clariflavum DSM 19732 possesses a broader array of hemicellulose-active enzymes compared to its close relative $C$. thermocellum as reported in its genome sequence [8], and preliminary exploration of the genome of strain 4-2a has revealed similar findings. Given that the rates of cellulose utilization in C. thermocellum and C. clariflavum strains are very similar (data not shown), it is possible to hypothesize that the expanded ability of $C$. clariflavum strains to break down hemicellulose may play a role in reducing inhibition of cellulases due to the presence of xylans and xylooligosaccharides $[17,18]$, which may potentially explain the increased ability of both $C$. clariflavum strains to break down the cellulose fraction in unpretreated switchgrass. However, in the case of all three strains, the breakdown of xylan only happens in the loosely associated layers (as detected in oxalate and carbonate fractions of glycome profiling) and not necessarily in the more difficult residual fractions, which likely accounts for the residual glucan, xylan, and arabinan detected through quantitative saccharification. Similar fractional solubilization of cellulose and hemicellulose was observed for all three Clostridia examined here, and has also been observed for C. bescii growing on unpretreated switchgrass [16]. Likewise, considering that these experiments were performed with unpretreated switchgrass, any kind of chemical pretreatment that enables access to these more recalcitrant residual fractions will help to overcome this limitation.

It should be noted that C. clariflavum and C. thermocellum both utilize a cellulosome for the breakdown of plant biomass $[8,19]$. Although cellulosomes provide a multitude of benefits for Clostridia growing on solid substrates, purified cellulosomes of $C$. thermocellum have been observed to have very low conversion rates in unpretreated switchgrass, as compared to uncomplexed enzymes [20]. Resch et al. [20] found that only $20 \%$ of glucan from unpretreated switchgrass was broken down by $C$. thermocellum cellulosomes, while we detect $42.5 \%$ glucan conversion with $C$. thermocellum and up to $68.7 \%$ in C. clariflavum str. $4-2 \mathrm{a}$. This discrepancy is possibly due to the fact that, under standard culture conditions, cellulosomal organisms also benefit from a large number of free cellulases, untethered cellulosomal structures, and tethered cellulosomes [8,21], as well as the continuous removal and utilization of released sugars.

\section{Conclusions}

In summary, the present study provides ample evidence of how $C$. clariflavum strains exhibit higher extents of hemicelluose breakdown than $C$. thermocellum given their ability to degrade xylan to xylooligomers and xylose, and, in 
the case of strain 4-2a, their ability to grow on xylose. This capability may also play a role in the higher levels of utilization of unpretreated plant material, as evidenced in total residual xylan composition and the fractions of easily accessible xylan utilized by these strains when growing on unpretreated switchgrass, as revealed by glycome profiling. Further exploration of other unique metabolic capabilities of C. clariflavum strains should be of great interest in the exploration and development of new CBP candidate organisms.

\section{Methods}

\section{Strains, media, and growth conditions}

Type cultures of C. clariflavum DSM 19732 and Clostridium thermocellum ATCC 27405 were obtained from DSM and ATCC, respectively. Clostridium clariflavum str. 4-2a was previously isolated from thermophilic compost enrichment cultures [2]. All growth experiments were conducted using defined MTC media, prepared as previously described [22]. For experiments using xylooligosaccharides as the sole carbon source, all experiments were conducted in serum tubes using small concentrations $(0.5$ to $0.8 \mathrm{~g} / \mathrm{L})$ of xylobiose, xylotriose, and xylotetraose (Megazyme, Bray, Ireland) with a total volume of $12 \mathrm{~mL}$ per experimental replicate. All other experiments were conducted in serum bottles with a total volume of $50 \mathrm{~mL}$ per experimental replicate. In experiments using xylose (Sigma) as the carbon source, substrate was added after filter sterilization to the other components of MTC. For experiments using solid substrates as the sole carbon source, triplicate serum bottles were prepared using either $3 \mathrm{~g} / \mathrm{L}$ birchwood xylan (Sigma) or switchgrass. Unpretreated switchgrass (Alamo, sieved 20/ 80 mesh fraction, grown in Athens, GA, harvested April 2011) was obtained from Dr. Ajaya Kumar Biswal and Dr. Debra Mohnen, University of Georgia, GA. Prior to its use in the growth experiments, the switchgrass was washed in consecutive washes with shaking until no soluble sugars were detected in the supernatant. Triplicate uninoculated control experiments were set up without any organisms and incubated at the same growth temperature as the experiments with inocula.

\section{Batch cultivation in fermentors}

For the switchgrass glycomics analyses, experiments were conducted in larger volumes in 1-liter Biostat Qplus fermentors (Sartorius Stedim, Gottingen, Germany), with a working volume of 1 liter and without $\mathrm{pH}$ control. The fermentors were equipped with Norprene tubing (Cole Palmer Instrument Company, Vernon Hills, IL) to minimize oxygen diffusion. Cultures were grown in the same MTC medium used for batch studies, with $3 \mathrm{~g}$ of switchgrass and with reducing agents and vitamins added separately after the autoclaving of fermentor vessels. Cultures were stirred at $100 \mathrm{rpm}$ and were sparged with $100 \mathrm{~mL} \mathrm{~min}^{-1}$ nitrogen gas. Each fermentation experiment was performed in separate duplicate fermentations and with a $5 \%(\mathrm{v} / \mathrm{v})$ inoculum of either C. thermocellum ATCC 27405, C. clariflavum DSM 19732, or C. clariflavum str. 4-2a. In addition, duplicate uninoculated reactors were run with switchgrass for the duration of the same inoculated experiments as uninoculated switchgrass controls. At the end of each 5-day fermentation period, residual plant material was washed, dried, weighed, and stored at $-80^{\circ} \mathrm{C}$ prior to the glycomics analyses.

\section{Analytical methods}

The concentrations of sugars and fermentation products (acetate, ethanol, formate, xylooligosaccharides, and xylose) were analyzed by high-performance liquid chromatography using an Aminex HPX-87H column (Bio-Rad, Hercules, CA) at $60^{\circ} \mathrm{C}$ with a refractive index (RI) detector and $2.5 \mathrm{mM}$ sulfuric acid as the mobile phase. Pellet nitrogen was measured in centrifuged pellet samples by using a TOC-V combustion analyzer coupled with a TNM-1 Total Nitrogen Module (Shimadzu Corporation, Columbia, MD) and comparing the results to a 1 -g liter ${ }^{-1}$ glycine standard as previously described [7]. Concentrations of glucan, xylan, and arabinan in residual switchgrass samples were determined using quantitative saccharification from residual material at the end of incubation, as described in Sluiter et al. [23].

\section{Switchgrass glycomics}

Glycome profiling analyses of switchgrass biomass residues were performed as described earlier [24,25], with increasingly harsh reagents $(50 \mathrm{mM}$ ammonium oxalate, $50 \mathrm{mM}$ sodium carbonate, $1 \mathrm{M}$ and $4 \mathrm{M}$ potassium hydroxide, and sodium chlorite in glacial acetic acid) used for the serial fractionation of plant cell walls and the thorough detection of all epitopes present in the switchgrass biomass residues. The resulting cell wall extracts were probed by ELISA using a comprehensive suite of 155 monoclonal antibodies directed against all major plant cell wall glycans (see Additional file 1: Table S1). Cell wall glycan-directed monoclonal antibodies were procured as hybridoma cell culture supernatants stocks from Complex Carbohydrate Research Center, University of Georgia, and are available from CarboSource Services [(www.carbosource.net); JIM, MAC and CCRC series]. Additional antibodies were purchased from Biosupplies Australia, Parkville, Victoria, Australia (LAMP and BG-1 antibodies) and were used as per the manufacturer's instructions. A detailed listing of all of the monoclonal antibodies used in this study is provided in Additional file 1: Table S1. 


\section{Additional file}

Additional file 1: Table S1. Listing of plant cell wall glycan-directed monoclonal antibodies (mAbs) used for glycome profiling analyses. The groupings of antibodies are based on a hierarchical clustering of ELISA data generated from a screen of all mAbs against a panel of plant polysaccharide preparations that groups the mAbs according to the predominant polysaccharides that they recognize. The majority of listings link to the WallMabDB plant cell wall monoclonal antibody database (http://www.wallmabdb.net) that provides detailed descriptions of each mAb, including immunogen, antibody isotype, epitope structure (to the extent known), supplier information, and related literature citations.

\section{Abbreviations}

CBP: consolidated bioprocessing; MTC: Medium for thermophilic Clostridia; $\mathrm{GH}$ : glycoside hydrolase.

\section{Competing interests}

LRL is a Co-founder and Director of Mascoma Corporation. All authors declare that they have no competing interests.

\section{Authors' contributions}

JAl designed the research approach, performed the research, and drafted the manuscript; SP performed the research and contributed to the drafting of the manuscript; AG provided research support; all authors analyzed or interpreted data, and provided important edits to various parts of the paper; JAI, SP, MGH, and LRL revised the overall paper; MGH and LRL supervised the work. All authors read and approved the final manuscript.

\section{Acknowledgements}

This work has been funded by BioEnergy Sciences Center (BESC), a U.S. Department of Energy (DOE) Bioenergy Research Center supported by the Office of Biological and Environmental Research in the DOE Office of Science. We would also like to thank Evert Holwerda and Julie Paye for useful technical discussions, and Debra Mohnen and Ajaya Biswal for providing the switchgrass material for this work.

\section{Author details}

${ }^{1}$ Thayer School of Engineering, Dartmouth College, Hanover, NH, USA ${ }^{2}$ BioEnergy Science Center Oak Ridge National Laboratory Oak Ridge, Oak Ridge, TN, USA. ${ }^{3}$ Department of Biology, Hofstra University, Hempstead, NY, USA. ${ }^{4}$ Complex Carbohydrate Research Center, University of Georgia, Athens, GA, USA.

Received: 5 June 2014 Accepted: 3 September 2014

Published online: 18 November 2014

\section{References}

1. Lynd L, Zyl W, McBride J, Laser M: Consolidated bioprocessing of cellulosic biomass: an update. Curr Opin Biotechnol 2005, 16:577-583.

2. Sizova MV, Izquierdo JA, Panikov NS, Lynd LR: Cellulose- and xylandegrading thermophilic anaerobic bacteria from biocompost. Appl Environ Microbiol 2011, 77:2282-2291.

3. Madden R: Isolation and characterization of Clostridium stercorarium sp. nov., cellulolytic thermophile. Int J Syst Bacterio/ 1983, 33:837.

4. Lynd L, Weimer P, Van Zyl W, Pretorius I: Microbial cellulose utilization: fundamentals and biotechnology. Microbiol Mol Biol Rev 2002, 66:506.

5. Shiratori H, Sasaya K, Ohiwa H, Ikeno H, Ayame S, Kataoka N, Miya A, Beppu T, Ueda K: Clostridium clariflavum sp. nov. and Clostridium caenicola sp. nov., moderately thermophilic, cellulose-/cellobiose-digesting bacteria isolated from methanogenic sludge. Int J Syst Evol Microbiol 2009, 59:1764-1770.

6. Shiratori $\mathrm{H}$, Ikeno $\mathrm{H}$, Ayame $\mathrm{S}$ : Isolation and characterization of a new Clostridium sp. that performs effective cellulosic waste digestion in a thermophilic methanogenic bioreactor. Appl Environ Microbiol 2006, 72:3702-3709.

7. Izquierdo JA, Sizova MV, Lynd LR: Diversity of bacteria and glycosyl hydrolase family 48 genes in cellulolytic consortia enriched from thermophilic biocompost. Appl Environ Microbiol 2010, 76:3545-3553.

8. Izquierdo JA, Goodwin L, Davenport KW, Teshima H, Bruce D, Detter C, Tapia R, Han S, Land M, Hauser L, Jeffries CD, Han J, Pitluck S, Nolan M,
Chen A, Huntemann M, Mavromatis K, Mikhailova N, Liolios K, Woyke T, Lynd LR: Complete genome sequence of Clostridium clariflavum DSM 19732. Stand Genomic Sci 2012, 6:104-115.

9. Gu Y, Ding Y, Ren C, Sun Z, Rodionov DA, Zhang W, Yang S, Yang C, Jiang $W$ : Reconstruction of xylose utilization pathway and regulons in Firmicutes. BMC Genomics 2010, 11:255

10. Zverlov W, Schwarz WH: Bacterial cellulose hydrolysis in anaerobic environmental subsystems - Clostridium thermocellum and Clostridium stercorarium, thermophilic plant-fiber degraders. Ann N Y Acad Sci 2008, 1125(Incredible Anaerobes from Physiology to Genomics to Fuels):298-307.

11. Taghavi S, Izquierdo JA, van der Lelie D: Complete genome sequence of Clostridium sp. strain DL-VIII, a novel solventogenic Clostridium species isolated from anaerobic sludge. Genome Announc 2013, 1(4):e00605-13.

12. Steen EJ, Kang Y, Bokinsky G, Hu Z, Schirmer A, Mcclure A, Cardayre SBD, Keasling JD: Microbial production of fatty-acid-derived fuels and chemicals from plant biomass. Nature 2010, 463:559-562.

13. Ho NW, Chen Z, Brainard AP, Sedlak M: Successful design and development of genetically engineered Saccharomyces yeasts for effective cofermentation of glucose and xylose from cellulosic biomass to fuel ethanol. Adv Biochem Eng Biotechnol 1999, 65:163-192.

14. Yang S-J, Kataeva I, Westpheling J, Adams MWW: Efficient degradation of lignocellulosic plant biomass, without pretreatment, by the thermophilic anaerobe "Anaerocellum thermophilum" DSM 6725. Appl Environ Microbiol 2009, 75:4762-4769.

15. Helle SS, Murray A, Lam J, Cameron DR, Duff SJB: Xylose fermentation by genetically modified Saccharomyces cerevisiae 259ST in spent sulfite liquor. Bioresour Technol 2004, 92:163-171.

16. Kataeva I, Foston MB, Yang S-J, Pattathil S, Biswal AK, Poole FL II, Basen M, Rhaesa AM, Thomas TP, Azadi P, Olman V, Saffold TD, Mohler KE, Lewis DL, Doeppke C, Zeng Y, Tschaplinski TJ, York WS, Davis M, Mohnen D, Xu Y, Ragauskas AJ, Ding S-Y, Kelly RM, Hahn MG, Adams MWW: Carbohydrate and lignin are simultaneously solubilized from unpretreated switchgrass by microbial action at high temperature. Energy Environ Sci 2013, 6:2186.

17. Kumar R, Wyman CE: Effect of xylanase supplementation of cellulase on digestion of corn stover solids prepared by leading pretreatment technologies. Bioresour Technol 2009, 100:4203-4213.

18. Shi J, Ebrik MA, Bin Y, Garlock RJ, Balan V, Dale BE, Pallapolu VR, Lee YY, Kim Y, Mosier NS, Ladisch MR, Holtzapple MT, Falls M, Sierra-Ramirez R, Donohoe BS, Vinzant TB, Elander RT, Hames B, Thomas S, Warner RE, Wyman CE: Application of cellulase and hemicellulase to pure xylan, pure cellulose, and switchgrass solids from leading pretreatments. Bioresour Technol 2011, 102:11080-11088.

19. Shoham $Y$, Lamed $R$, Bayer E: The cellulosome concept as an efficient microbial strategy for the degradation of insoluble polysaccharides. Trends Microbiol 1999, 7:275-281.

20. Resch MG, Donohoe BS, Baker JO, Decker SR, Bayer EA, Beckham GT, Himmel ME: Fungal cellulases and complexed cellulosomal enzymes exhibit synergistic mechanisms in cellulose deconstruction. Energy Environ Sci 2013, 6:1858.

21. Mohand-Oussaid O, Payot S, Guedon E, Gelhaye E, Youyou A, Petitdemange $\mathrm{H}$ : The extracellular xylan degradative system in Clostridium cellulolyticum cultivated on xylan: evidence for cell-free cellulosome production. J Bacteriol 1999, 181:4035-4040.

22. Holwerda EK, Hirst KD, Lynd LR: A defined growth medium with very low background carbon for culturing Clostridium thermocellum. J Ind Microbiol Biotechnol 2012, 39(6):943-947.

23. Sluiter A, Hames B, Ruiz R, Scarlata C, Sluiter J, Templeton D, NREL ADC: 42618, Technical Report NREL/TP-510-42618. 2011:1-18.

24. Pattathil S, Avci U, Baldwin D, Swennes AG, McGill JA, Popper Z, Bootten T, Albert A, Davis RH, Chennareddy C, Dong R, O'Shea B, Rossi R, Leoff C, Freshour G, Narra R, O'Neil M, York WS, Hahn MG: A comprehensive toolkit of plant cell wall glycan-directed monoclonal antibodies. Plant Physiol 2010, 153:514-525.

25. Pattathil S, Avci U, Miller JS, Hahn MG: Immunological approaches to plant cell wall and biomass characterization: glycome profiling. Methods $\mathrm{Mol}$ Biol 2012, 908:61-72.

\section{doi:10.1186/s13068-014-0136-4}

Cite this article as: Izquierdo et al:: Comparative analysis of the ability of Clostridium clariflavum strains and Clostridium thermocellum to utilize hemicellulose and unpretreated plant material. Biotechnology for Biofuels 2014 7:136. 